\title{
Roles of MicroRNA-122 in Cardiovascular Fibrosis and Related Diseases
}

\author{
Ying Liu ${ }^{1,2} \cdot$ Jia-Wei Song ${ }^{1} \cdot$ Jian-Yu Lin ${ }^{3} \cdot$ Ran Miao $^{1,2} \cdot$ Jiu-Chang Zhong ${ }^{1,2}$ (1)
}

Received: 25 June 2020 / Accepted: 18 August 2020 / Published online: 27 August 2020

(c) Springer Science+Business Media, LLC, part of Springer Nature 2020

\begin{abstract}
Fibrotic diseases cause annually more than 800,000 deaths worldwide, where of the majority accounts for cardiovascular fibrosis, which is characterized by endothelial dysfunction, myocardial stiffening and reduced dispensability. MicroRNAs (miRs), small noncoding RNAs, play critical roles in cardiovascular dysfunction and related disorders. Intriguingly, there is a critical link among miR-122, cardiovascular fibrosis, sirtuin 6 (SIRT6) and angiotensin-converting enzyme 2 (ACE2), which was recently identified as a coreceptor for SARS-CoV2 and a negative regulator of the rennin-angiotensin system. MiR-122 overexpression appears to exacerbate the angiotensin II-mediated loss of autophagy and increased inflammation, apoptosis, extracellular matrix deposition, cardiovascular fibrosis and dysfunction by modulating the SIRT6-Elabela-ACE2, LGR4- $\beta$-catenin, TGF $\beta$-CTGF and PTEN-PI3K-Akt signaling pathways. More importantly, the inhibition of miR-122 has proautophagic, antioxidant, anti-inflammatory, anti-apoptotic and antifibrotic effects. Clinical and experimental studies clearly demonstrate that miR-122 functions as a crucial hallmark of fibrogenesis, cardiovascular injury and dysfunction. Additionally, the miR-122 level is related to the severity of hypertension, atherosclerosis, atrial fibrillation, acute myocardial infarction and heart failure, and miR-122 expression is a risk factor for these diseases. The miR-122 level has emerged as an early-warning biomarker cardiovascular fibrosis, and targeting miR-122 is a novel therapeutic approach against progression of cardiovascular dysfunction. Therefore, an increased understanding of the cardiovascular roles of miR-122 will help the development of effective interventions. This review summarizes the biogenesis of miR-122; regulatory effects and underlying mechanisms of miR-122 on cardiovascular fibrosis and related diseases; and its function as a potential specific biomarker for cardiovascular dysfunction.
\end{abstract}

Keywords microRNA-122 · Fibrosis · Cardiovascular dysfunction $\cdot$ Sirtuin $6 \cdot$ ACE2

\section{Introduction}

Cardiovascular fibrosis, a vital cause of heart failure (HF), refers to the development of scar tissue in the injured heart and blood vessels due to an aberrant wound-healing response

Communicated by Kurt J. Varner.

Ran Miao

mr1019@163.com

Jiu-Chang Zhong

jczhong@sina.com

1 Heart Center and Beijing Key Laboratory of Hypertension, Beijing Chaoyang Hospital Affiliated to Capital Medical University, Beijing 100020, China

2 Medical Research Center, Beijing Chaoyang Hospital, Capital Medical University, Beijing 100020, China

3 Department of Comprehensive Surgery, Beijing Chaoyang Hospital, Capital Medical University, Beijing 100020, China to injury or insult, and arises from enhanced inflammatory processes, uncontrolled cell proliferation and excessive oxidative stress [1-3]. Established fibrosis is a hallmark of chronic disease progression and a key contributor to cardiovascular stiffness and injury of the heart and vessels, ultimately leading to HF and vascular dysfunction [1-4]. Since wound healing and fibrotic responses to myocardial and vascular injury are multifactorial processes, current therapies that specifically target factors that contribute to only cardiovascular disease pathogenesis offer limited overall anti-fibrotic efficacy [3]. Fibrosis-induced cardiovascular remodeling and injury are growing problems for improving strategies to prevent the development of fibrosis and reduce the mortality of HF [1-3].

MicroRNAs (miRs) are non-protein-encoding RNAs with a length of 21-25 bases, that degrade mRNA or bind the 3' noncoding regions (UCRs) of their target genes with incomplete complementarity and regulate gene expression at the 
posttranscriptional level $[1,5,6]$. MiR-122 may be a sensitive and strong predictor of cardiovascular injury and failure in patients with fibrosis. Although the biological functions of miR-122 are still largely unknown, miR-122 has been shown to regulate cardiovascular inflammation, autophagy, apoptosis, oxidative stress, fibrosis and dysfunction $[1,5$, 7-9]. MiR-122 exerts different and even opposing regulatory effects on the cardiovascular system and binds its target genes to control the levels of pro-inflammatory and fibrotic factors [1, 5-7]. Notably, miR-122 functions as a risk biomarker of cardiovascular fibrosis and appears to be a direct participant in the development of cardiovascular diseases, including hypertension, atherosclerosis, HF, myocardial infarction (MI) and atrial fibrillation (Fig. 1) [1, 5-9]. In this review, we highlight the developments and latest advances concerning the biogenesis and biological effects of miR-122 signaling and the regulatory roles of miR-122 in cardiovascular fibrosis and dysfunction.

\section{Biogenesis and Biological Effects of MiR-122}

MiR-122 is located on chromosome 18, from which the $\sim 4.5 \mathrm{~kb}$ pre-miR-122 is generated, after which premiR-122 is processed to mature miR-122, which moves from the nucleus to the cytoplasm [7]. The promoter region of miR-122 is highly conserved and located approximately $5 \mathrm{~kb}$ upstream of the conserved stem-loop sequences in miR122 , which include large nuclear receptor factor-binding sequences, such as the TATA-box and CCAAT-box [8]. Notably, sirtuin 6 (SIRT6), a miR-122 target gene, is important regulator of cardiovascular fibrosis, remodeling and dysfunction [1]. Intriguingly, SIRT1, SIRT6, Apelin (APLN), Apelin receptor (APLNR) and forkhead box O3 (FOXO3) were identified as miR-122 target genes (Fig. 2). The predicted interactions between miR-122 and the abovementioned targets were analyzed through the miRs target gene prediction website (https://www.microrna.org). Both miR-122-3p and miR-122-5p are widely expressed in endothelial cells (ECs), cardiomyocytes (CMs), cardiac fibroblasts (CFs), adipocytes, vascular smooth muscle cells (VSMCs) and adventitial fibroblasts (AFs) in the cardiovascular system (Table 1; Fig. 3) $[1,6,9,10]$. Recently, administration of a miR-122 inhibitor was shown to reduce the key transforming growth factor- $\beta$ (TGF- $\beta$ )-induced fibrotic signaling pathway, promotes collagen synthesis and stimulates fibrinogenesis, consequently resulting in the accumulation of fibroblasts and extracellular matrix (ECM), in hypertension and cardiovascular diseases $[2,3,9,11]$. In our previous study, we observed cardiovascular fibrosis, systolic dysfunction, cardiovascular remodeling and dysfunction in rats after transverse aortic constriction (TAC) or angiotensin II (Ang II) stimulation, and these

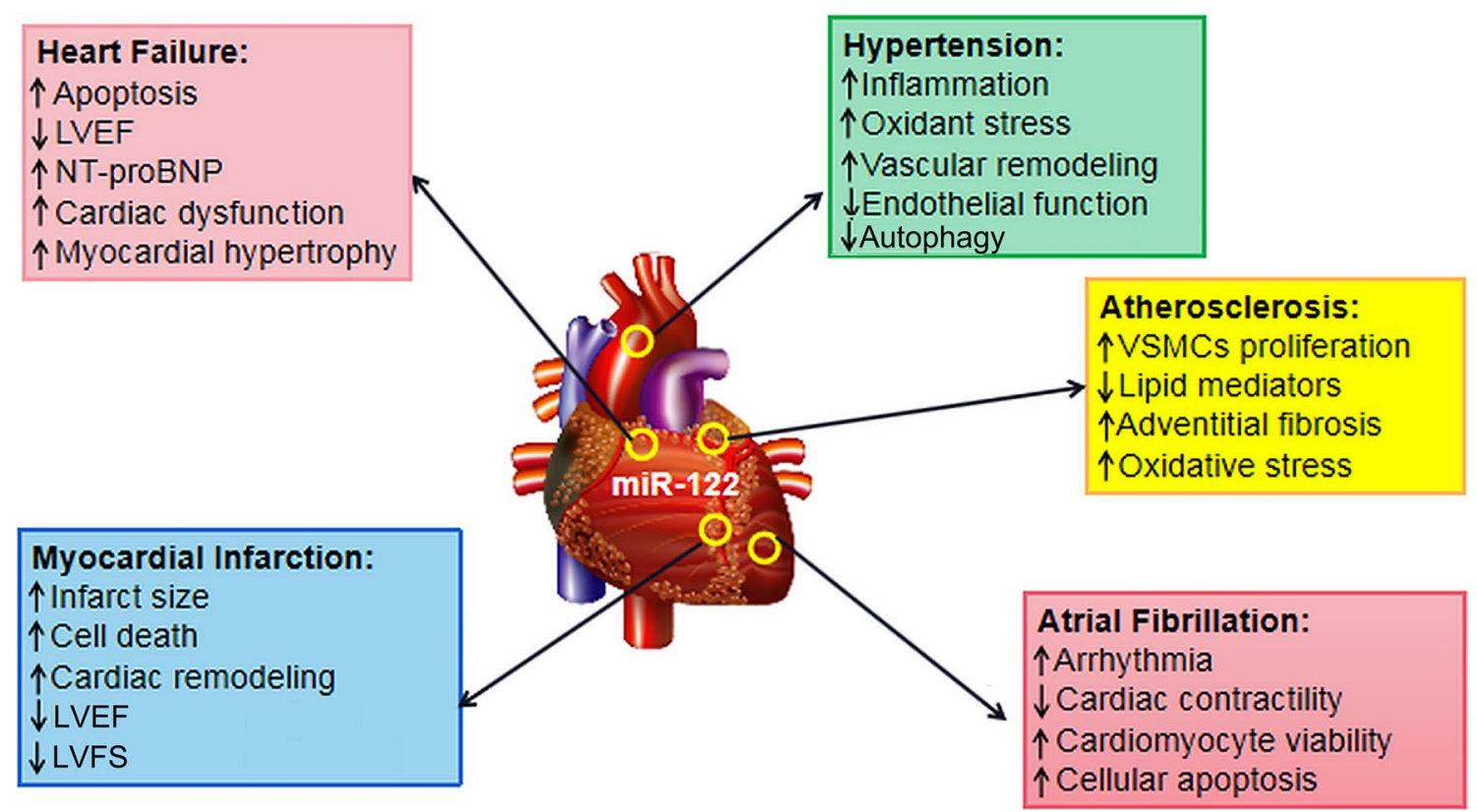

Fig. 1 Central roles of miR-122 in HF, hypertension, MI, atherosclerosis and atrial fibrillation. MiR-122 has been shown to promote apoptosis, inflammation, fibrosis, pathological hypertrophy and remodeling in the cardiovascular system; decrease the LVEF, LVFS and cardiac contractility; and increase NT-proBNP and ROS generation, leading to arrhythmia and cardiovascular dysfunction. There- fore, miR-122 can cause cardiovascular fibrosis and heart dysfunction, ultimately resulting in hypertension, atherosclerosis, MI and HF. $M I$ myocardial infarction, $H F$ heart failure, $R O S$ reactive oxygen species, $L V F S$ left ventricular fractional shortening, $L V E F$ left ventricular ejection fraction 


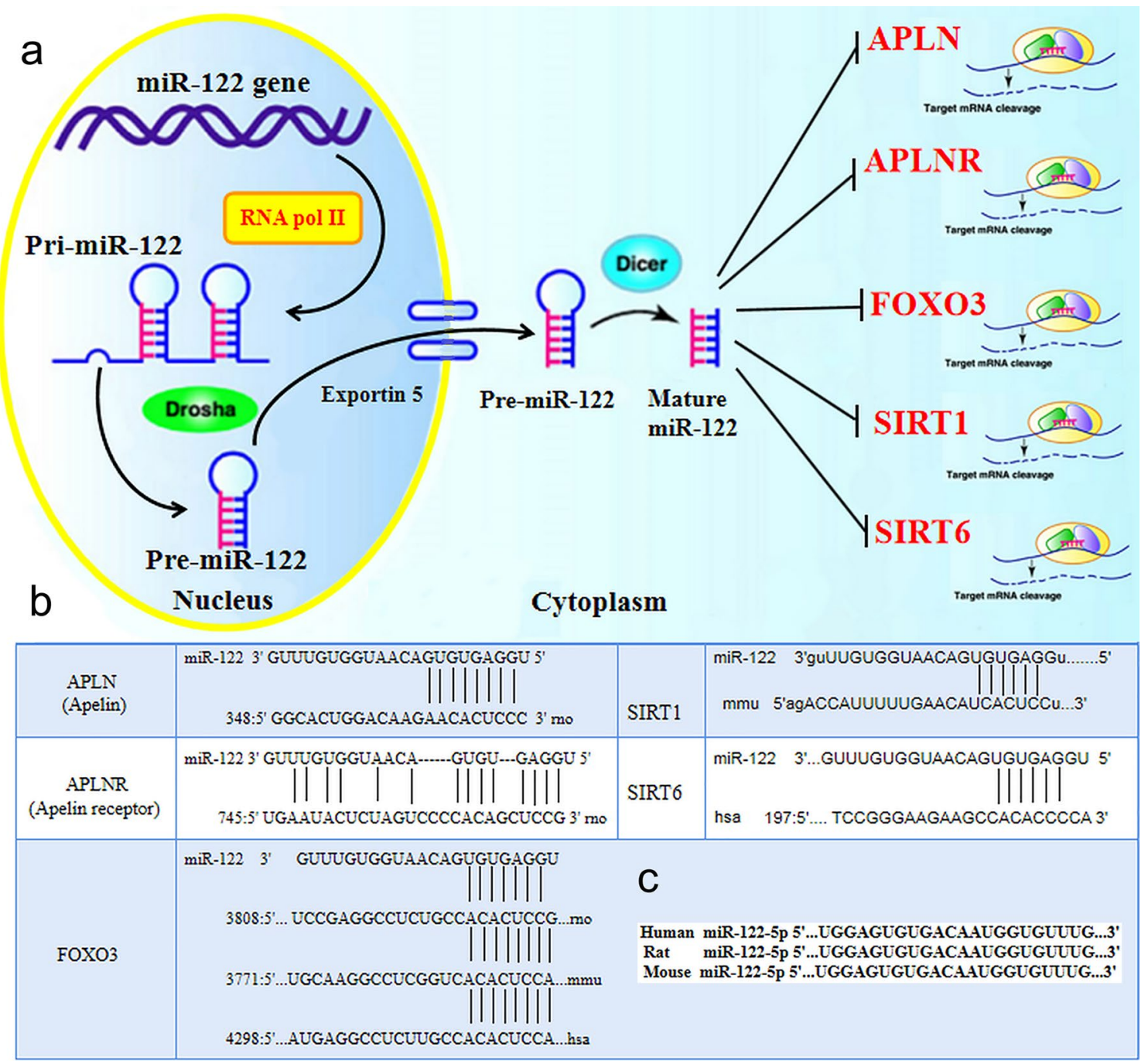

Fig. 2 Schematic diagram of the activities, and target genes of miR122 and potential miR-122-binding sites in these target genes. a Schematic diagram showing the mechanism of miR-122. b APLN, APLNR, SIRT1, SIRT6 and FOXO3 were identified as miR-122 target genes by the use of the publicly available bioinformatics tool Microcosm Targets and microRNA.org. The predicted interactions

effects were associated with increased levels of miR-122-5p, and reduced levels of SIRT6, elabela (ELA), angiotensin-converting enzyme 2 (ACE2) and phosphorylated adenosine 5 '-monophosphate-activated protein kinase (AMPK) (Table 1) $[1,2,6]$. In uric acid-treated HK-2 tubular epithelial cells, treatment with miR-122 mimics markedly increased NLRP3 inflammasome activation by increasing the levels of BRCA1/ BRCA2-containing complex 3 (Table 1) [12]. Intriguingly, miR-122-5p was found to accelerate ischemic reperfusion (I/R) injury-induced renal damage, through increased fibrosis, inflammation, and apoptosis, and promote renal dysfunction [13]. In a rat model of renal I/R injury, supplementation with a miR-122 inhibitor prevented apoptosis and reactive oxygen species (ROS) generation through modulating phosphatase and between miR-122 and the abovementioned targets were shown and analyzed with a miR target gene prediction website (https://www. microrna.org). c The sequence of miR-122 is highly conserved in humans, mice and rats. FOXO3 forkhead box O3, SIRT1 sirtuin 1, SIRT6 sirtuin 6, APLN Apelin, APLNR Apelin receptor

tensin homolog (PTEN)/ phosphoinositide 3-kinase (PI3K)/ Akt pathway activity (Table 1; Fig. 3) [13]. Conversely, levels of the proinflammatory cytokines tumor necrosis factor- $\alpha$ $(\mathrm{TNF}-\alpha)$ and the number of macrophages $\left(\mathrm{F} 4 / 80^{+} \mathrm{CD} 11 \mathrm{~b}^{+}\right)$ were reduced in miR-122 knockout (KO) mice, suggesting that miR-122 deficiency inhibited inflammation and tissue damage [14]. In L02 cells pretreated with oleic acid, treatment with a miR-122 inhibitor mitigated lipid accumulation and inflammation through repressing the TLR4/myeloid differentiation primary response gene 88 (MyD88)/ nuclear factor-кB (NFкB) p65 pathway (Table 1) [15]. MiR-122 levels were elevated in a mouse model of lung fibrosis, and this increase was linked with enhanced inflammation and pulmonary vascular remodeling [16]. Importantly, miR-122 functions as a predictive 
Table 1 The regulatory roles and underlying mechanisms of miR-122 in cardiovascular fibrosis, dysfunction and related diseases

\begin{tabular}{|c|c|c|c|}
\hline Experimental model & Experimental intervention & Effects & References \\
\hline Rat AFs pretreated with Ang II & miR-122 mimic & $\begin{array}{l}\downarrow \text { SIRT6, ELA, ACE2 level } \\
\downarrow \text { Beclin-1, LC3II, autophagy } \\
\uparrow \text { P62, p-mTOR } \\
\uparrow \text { Cellular migration, oxidative stress }\end{array}$ & {$[1]$} \\
\hline Rat AFs pretreated with Ang II & miR-122 inhibitor & $\begin{array}{l}\uparrow \text { p-AMPK, LC3II } \\
\downarrow \text { P62, p-mTOR; IL-18, IL-33 } \\
\uparrow \text { Autophagy } \\
\downarrow \text { Apoptosis, ROS production }\end{array}$ & {$[1]$} \\
\hline Rats with TAC & $\begin{array}{l}\text { Apelin-13 or } \\
\text { Irbesartan }\end{array}$ & $\begin{array}{l}\downarrow \text { miR-122 levels } \\
\downarrow \text { TGF } \beta 1 / \text { CTGF/collagen I/III } \\
\downarrow \text { aortic fibrosis }\end{array}$ & {$[6]$} \\
\hline Rat AFs pretreated with Ang II & miR-122 inhibitor & $\begin{array}{l}\downarrow \text { TGF } \beta 1 / \text { CTGF } \\
\downarrow \text { LGR } 4 / \beta \text {-catenin } \\
\downarrow \text { Apoptosis, cellular migration }\end{array}$ & {$[6]$} \\
\hline Patients with $\mathrm{HF}$ and $\mathrm{CKD}$ & - & $\begin{array}{l}\uparrow \mathrm{miR}-122 \text { levels } \\
\uparrow \mathrm{TGF}-\beta 1 \\
\uparrow \text { renal fibrosis }\end{array}$ & [9] \\
\hline Renal tubular epithelial HK-2 cells & miR-122 mimics & $\begin{array}{l}\uparrow N L R P 3 \text { inflammasome } \\
\uparrow \text { Inflammation }\end{array}$ & [12] \\
\hline SD rats with renal ischemia-reperfusion & miR-122 inhibitor & $\begin{array}{l}\uparrow \mathrm{PTEN} / \mathrm{PI} 3 \mathrm{~K} / \mathrm{Akt} \\
\downarrow \mathrm{ROS} \text { production }\end{array}$ & [13] \\
\hline MiR-122KO mice & - & $\begin{array}{l}\uparrow \mathrm{TNF}-\alpha \\
\uparrow \mathrm{F} 4 / 80^{+} \mathrm{CD} 11 \mathrm{~b}^{+}\end{array}$ & [14] \\
\hline L02 cells pretreated by oleic acid & miR-122 inhibitor & $\begin{array}{l}\downarrow \text { TLR4/MyD88 } \\
\downarrow \text { NF-кB p65 }\end{array}$ & {$[15]$} \\
\hline Ang II induced hypertensive mice & - & $\begin{array}{l}\uparrow \mathrm{miR}-122, \text { Bach-1, MCP-1 } \\
\downarrow \text { HO- } 1 \\
\uparrow \text { Collagen deposition and fibrosis }\end{array}$ & {$[17]$} \\
\hline NRVMs & miR-122 mimics & $\begin{array}{l}\downarrow \text { FoxO3 } \\
\uparrow \text { Calcineurin, ANP, BNP } \\
\uparrow \text { hypertrophy, remodeling }\end{array}$ & [18] \\
\hline Younger patients with hypertension & - & $\begin{array}{l}\uparrow \mathrm{miR}-122 \text { levels } \\
\uparrow \text { Endothelial dysfunction } \\
\downarrow \text { CAT-1 }\end{array}$ & [19] \\
\hline Patients with BAV & - & $\begin{array}{l}\uparrow \text { miR-122,TGF- } \beta 1 \text {, VEGF levels } \\
\uparrow \text { Valvular dysfunction } \\
\uparrow \text { Apoptosis; ECM remodeling }\end{array}$ & {$[20]$} \\
\hline Patients with HF & - & $\begin{array}{l}\uparrow m i R-122, \text { NT-proBNP levels } \\
\uparrow \text { Heart dysfunction }\end{array}$ & {$[21]$} \\
\hline Ligation of LAD in SD rats & Anti-miR-122 & $\begin{array}{l}\downarrow \text { Infarct Size } \\
\uparrow \text { LVFS; LVEF }\end{array}$ & {$[22]$} \\
\hline OGD-induced $\mathrm{H} 9 \mathrm{C} 2$ cells & miR-122 mimics & $\begin{array}{l}\uparrow \text { AKT/GSK-3 } \beta / \beta \text {-catenin } \\
\downarrow \text { Apoptosis, epicardial expansion } \\
\downarrow \text { Epithelial-mesenchy } \\
\uparrow \text { Cardiac dysfunction }\end{array}$ & {$[23]$} \\
\hline OGD-induced $\mathrm{H} 9 \mathrm{C} 2$ cells & miR-122 inhibitor & $\begin{array}{l}\uparrow J N K / p 38 \text { MAPK } \\
\downarrow \text { mTOR; Apoptosis } \\
\uparrow \text { Cell viability }\end{array}$ & {$[24]$} \\
\hline Mice with atrial fibrillation & miR-122 inhibitor & $\begin{array}{l}\uparrow \text { Bcl-x } \\
\downarrow \text { Caspase-3; p-ERK1/2 }\end{array}$ & {$[25]$} \\
\hline
\end{tabular}

$A C E 2$ angiotensin converting enzyme 2, AMPK adenosine 5'-monophosphate-activated protein kinase, $A F s$ adventitial fibroblasts, $C F s$ cardiofibroblasts, $C K D$ chronic kidney disease, ERK extracellular regulated protein kinases, HF heart failure, KO knockout, SIRT6 sirtuin 6, ELA elabela, $O G D$ oxygen-glucose deprivation, $N L R P 3$ nod-like receptor protein 3, LGR4 leucine-rich repeat-containing G protein-coupled receptor 4, GSK-3 $\beta$ glycogen synthase kinase- $3 \beta$ antibody, PTEN gene of phosphate and tension homology deleted on chromosome ten, PI3K phosphatidylinositol 3-kinase, Akt serine/threonine protein kinase, CTGF connective tissue growth factor, $m T O R$ mammalian target of rapamycin, NRVMs neonatal rat ventricular cardiomyocytes, TAC transverse aortic constriction, TGF- $\beta 1$ transforming growth factor- $\beta 1, F O X O 3$ forkhead box O3, Bach-1 BTB and CNC homology 1, HO-1 heme oxygenase1, MCP-1 monocyte chemotactic protein 1, TNF- $\alpha$ tumor necrosis factor $\alpha, L A D$ left anterior descending artery, $L V F S$ left ventricular fractional shortening, $L V E F$ left ventricular ejection fraction, $R O S$ reactive oxygen species 


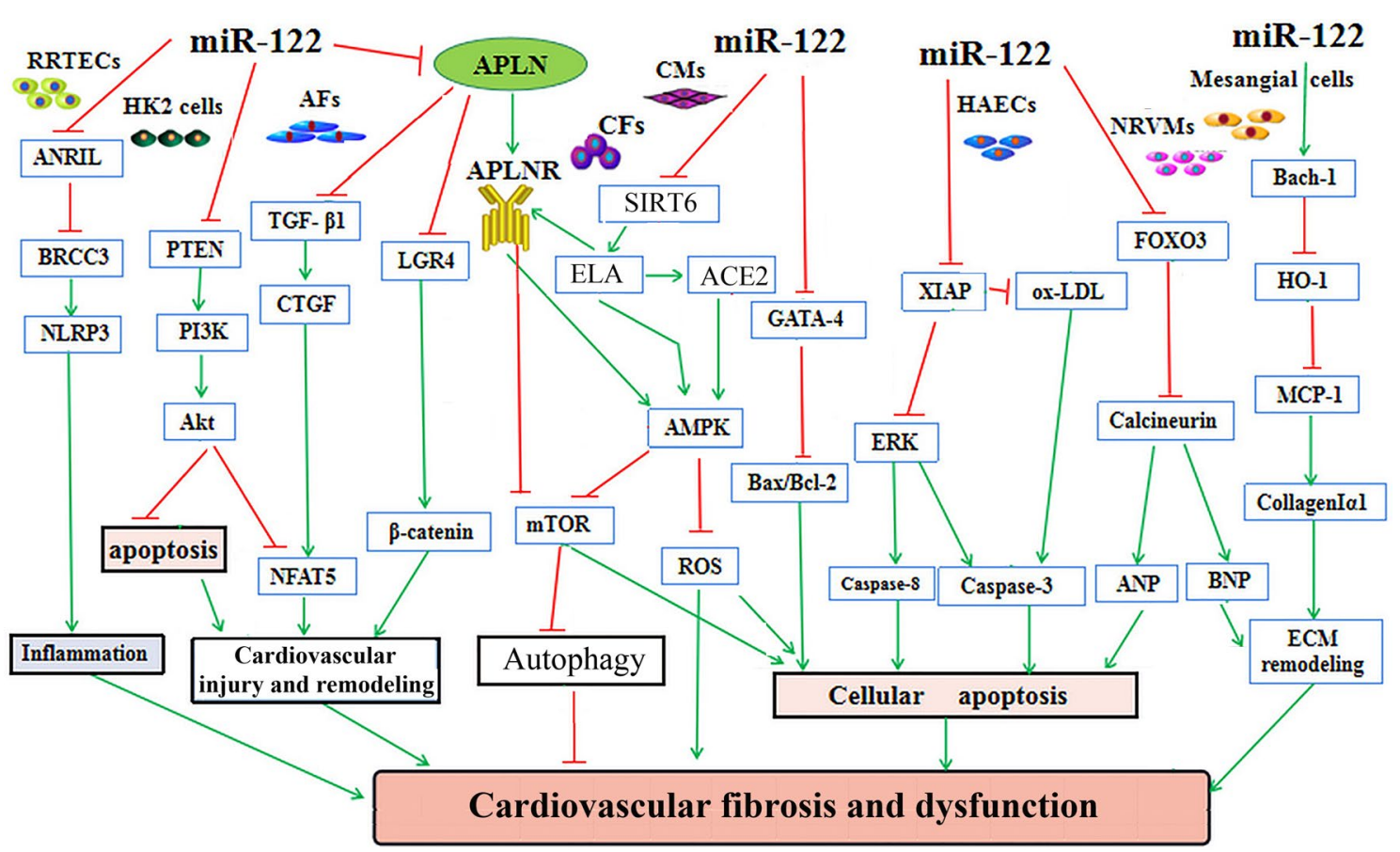

Fig. 3 The regulatory roles and underlying mechanisms of miR-122 in cardiovascular remodeling, fibrosis and dysfunction. MiR-122 plays a role in regulating cell growth, survival, inflammation, ECM deposition, pathological remodeling, cardiovascular fibrosis and dysfunction in RRTECs, HAECs, NRVMs, CMs, AFs, CFs, and HK2 cells by modulating the ANRIL-BRCC3, FOXO3-Calcineurin, Bach-1/HO-1, TGF $\beta$-CTGF-NFAT5 and PTEN-PI3K-Akt signaling pathways, respectively. Furthermore, the inhibition of miR-122 has been shown to modulate cardiac contractility, autophagy, apoptosis, and oxidative stress by regulating of the SIRT6-ELA-ACE2, GATA4-Bax, XIAP-ERK-Caspase, and LGR4- $\beta$-catenin signaling, respectively. ACE2 angiotensin-converting enzyme 2, AFs adventitial fibrotic cells, CFs cardiofibroblasts, CMs cardiomyocytes, HAECs, Human aortic endothelial cells, NRVMs neonatal rat cardiomyocytes, RRTECs rat renal tubular epithelial cells, HK2 cells human renal tubular epithelial cells, ANRIL antisense non-coding RNA in the INK4 locus, BRCC3 BRCA1/BRCA2-containing complex subunit 3, NLRP3nod-like receptor protein 3, PTEN gene of phosphate and tension homology deleted on chromosome ten, $P I 3 K$ phosphatidylinositol 3-kinase, TGF- $\beta$ transforming growth factor- $\beta, C T G F$ connective tissue growth factor, NFAT5 nuclear factor of activated T-cell-5, $L G R 4$ leucine-rich repeat-containing $\mathrm{G}$ protein-coupled receptor 4, $R O S$ reactive oxygen species, mTOR mammalian target of rapamycin, ECM extracellular matrix, $A M P K$ adenosine 5 '-monophosphateactivated protein kinase, GATA4 GATA binding protein 4, SIRT6 sirtuin 6, ELA elabela, ERK extracellular signal-regulated kinase, XIAP $\mathrm{X}$-linked inhibitor of apoptosis protein, $\mathrm{FOXO3}$ forkhead box O3, Bach-1 BTB and CNC homology 1, HO-1 heme oxygenase1, MCP-1 monocyte chemotactic protein 1 marker of fibrosis and appears to stimulate the inflammation and oxidative stress in the heart, blood vessels and liver [1, 6, $8,11,15]$. MiR-122 has been found to increased levels of the fibrotic factors collagen $1 \alpha 1$, collagen $1 \alpha 2$ and TGF- $\beta 1$ and the proinflammatory factor monocyte chemoattractant protein-1 (MCP-1) [15]. Collectively, these results reveal that miR$122-5$ p plays critical roles in the controlling cardiovascular fibrosis, inflammation, apoptosis, myocardial remodeling and dysfunction by activating the TGF $\beta 1$-CTGF, MyD88/NF-кB and PTEN/PI3K/Akt signaling pathways (Fig. 3).

\section{MiR-122, and Cardiovascular Fibrosis and Remodeling}

Cardiovascular fibrosis contributes to cardiac or arterial stiffening, endothelial dysfunction, wall thickening, reduced dispensability, and coronary atherosclerotic stenosis, leading to hemodynamic damage, the destruction of cardiovascular tissues and HF [2, 26-29]. A variety 
of alterations in the interstitial myocardial collagen network, macrophages, CMs, CFs, ECs and vascular cells are closely linked with fibrillar alteration in the heart. At the molecular and cellular levels, cardiovascular fibrosis is linked with reduced nitric oxide release, increased ROS generation, the activation of transcription factors, the stimulation of proinflammatory and profibrotic pathways, increased collagen deposition and ECM remodeling [1, 3, 28-31]. Inflammation and oxidative stress seem to be positively associated with remodeling processes, ultimately contributing to cardiac dilation and dysfunction [13, 29]. MiRs participate in regulating cell proliferation, migration, differentiation, and apoptosis and function as crucial determinants and biomarkers of cardiovascular fibrosis [1, $5,6,30,32]$. In particular, miR-122 has been implicated in the progression of fibrosis and acts as a circulatory biomarker in hypertension and $\mathrm{HF}$ [1, 6, 17, 18, 33-37]. We previously demonstrated that miR-122 overexpression exacerbated the loss of autophagy and increased cellular migration, apoptosis, extracellular matrix deposition mediated by angiotensin II by modulating the SIRT6-ELAACE2, leucine-rich repeat-containing G-protein-coupled receptors 4 (LGR4)- $\beta$-catenin, and TGF $\beta$-CTGF signaling pathways (Table 1; Fig. 3) [1, 6], indicating that miR-122 inhibition is a promising therapeutic strategy for cardiac fibrosis and dysfunction. Surprisingly, the levels of circulating exosomal miR-122 were positively associated with cardiac dysfunction in patients with HF with a reduced left ventricle (LV) ejection fraction (EF) and elevated levels of NT-proBNP [36]. A recent study demonstrated that miR122 was highly expressed in cultured $\mathrm{H} 9 \mathrm{C} 2$ cells under hypoxia/reoxygenation, and overexpression of miR-122 by recombinant adeno-associated infection significantly augmented the apoptosis of $\mathrm{H} 9 \mathrm{C} 2$ cells [37]. In contrast, inhibition of miR-122 attenuated pathological cardiac remodeling and decreased cell apoptosis in myocardial ischemic injury [37]. In cultured neonatal rat ventricular myocytes (NRVMs), overexpression of miR-122 by miR-122 mimics increased the size of CMs and hypertrophic gene expression, but downregulated the expression of anti-hypertrophic genes upon Ang II stimulation (Table 1) [18]. Intriguingly, bioinformatics analysis and luciferase reporter assays revealed that miR-122 directly targets FoxO3 (Fig. 2). Moreover, miR-122 was found to decrease FoxO3 levels but promote activation of the calcineurin signaling pathway, implying that miR-122 accelerates the development of cardiovascular hypertrophy and remodeling via modulation of the FoxO3-calcineurin pathway (Table 1; Fig. 3) [18]. Thus, miR-122 inhibition might be a promising field for the therapeutic approach in cardiovascular fibrosis and related diseases.

\section{Roles of MiR-122 in Hypertension}

Hypertension is a complex cardiovascular syndrome, while its pathogenesis is closely related to abnormalities in miRs and the renin-angiotensin system (RAS) $[1,6,17$, $18,32,36-39]$. MiR-122 plays a key role in the regulation of cardiovascular fibrosis and endothelial function during hypertension (Table 1) [1, 17, 18, 37, 38]. Intriguingly, stimulation with Ang II markedly increased the expression of miR-122 in NRVMs [36]. Our previous study demonstrated that administration of a miR-122 inhibitor effectively prevented the loss of autophagy and increased cellular proliferation, migration, apoptosis and cardiovascular fibrosis induced by Ang II via modulation of the SIRT6ELA-ACE2, LGR4/ $\beta$-catenin and TGF $\beta 1$-CTGF-NFAT5 signaling pathways (Table 1; Fig. 3) [1, 6]. Intriguingly, miR-122 was identified as an independent predictor of renovascular hypertension linked with increased collagen deposition and cardiovascular fibrosis. Administration of a miR-122 mimic led to a marked increase in BTB and $\mathrm{CNC}$ homology 1 transcription factor (Bach-1) expression in mesangial cells [17]. In contrast, inhibition of miR-122 blunted expression of the proinflammatory factors Bach-1 and MCP-1 and the profibrotic factor collagen $1 \alpha 1$ in aged mice with Ang II-induced hypertension (Table 1; Fig. 3) [17]. These data suggested a crosstalk between miR-122 and the RAS in the control of fibrosis, inflammation and remodeling during hypertension.

MiR-122 has been implicated in occurrence and development of hypertension and HF [1, 6, 19, 20, 40-42]. Elevated levels of plasma miR-122 were found in patients with essential hypertension, especially younger patients with hypertension [19]. Overexpression of miR-122 resulted in endothelial dysfunction by suppressing the expression of cationic amino acid transporter 1, which destroyed the endothelial structure, increasing risk factors for cardiovascular dysfunction during hypertension (Table 1; Fig. 3) [19]. MiR-122 levels were also significantly upregulated in patients with hypertension, indicating the role of miR-122 as predictive hazard factor in cardiovascular homeostasis and disorder [19, 20, 40-42]. MiR-122 plays a critical role in the development of the bicuspid aortic valve (BAV) and aortic dilation by promoting the apoptosis of ECs and cardiovascular remodeling via the activation of TGF- $\beta$-VEGF signaling pathways (Table 1; Fig. 3) [20]. Intriguingly, bioinformatics analysis and luciferase reporter assays revealed that miR-122 directly targets SIRT6 and attenuates the level of SIRT6 (Fig. 2) [43]. Our previous work revealed that SIRT6 overexpression strikingly promotes cardiac levels of pAMPK $\alpha$ and ACE2 and decreases fibrotic gene levels of CTGF, TGF $\beta 1$, collagen I and collagen III, leading to alleviation of Ang 
II-induced pathological hypertrophy, myocardial fibrosis, cardiac ultrastructural injury and dysfunction in hypertensive rats [44]. MiR-122, which is expressed in a variety of cardiovascular system cell types, can regulate gene expression at the posttranscriptional level and plays an important role in the occurrence and development of cardiovascular diseases (Fig. 1). Furthermore, miR-122 was implicated in cardiorenal injury and vascular remodeling in spontaneously hypertensive in African green monkey and rat models of renovascular hypertension by regulating lipoprotein metabolism and cholesterol homeostasis [45, 46]. Taken together, these findings show that the inhibition of miR-122 exerts protective effects against cardiovascular fibrosis and dysfunction during hypertension.

\section{Roles of MiR-122 in Atherosclerosis}

Atherosclerosis is a chronic inflammatory vascular disease characterized with abnormal cholesterol metabolism, an abnormal inflammatory response, endothelial dysfunction, apoptosis and VSMC proliferation [11, 20, 47]. MiR-122 is a well-known marker of cardiovascular events and a good predictor of atherosclerosis. MiR-122 levels were shown to be upregulated in patients with atherosclerotic lesions, and serum miR-122 levels were positively correlated with atherosclerotic severity [20, 48, 49]. In fact, miR-122 promotes proinflammatory factors and oxidant injury in the liver and cardiovascular system $[10,12,13]$. Thus, potential usage of miR-122 antagonism in treatment of inflammatory injury may represent a novel therapeutic approach for atherosclerosis. Moreover, apoptosis in ECs is the basis of pathophysiology of atherosclerosis [13, 45, 49]. In apolipoprotein E deficient mice fed a high-fat diet, miR-122 expression was increased in aortic ECs. In addition, miR-122 inhibitor strikingly suppressed ox-LDL-induced apoptosis of human aortic ECs, suggesting the anti-apoptotic role of miR-122 inhibition under pro-atherogenic conditions [49]. Further evidence revealed that the X-linked inhibitor-of-apoptosis protein (XIAP) is directly targeted and suppressed by miR122 in ECs. More importantly, XIAP knockdown diminished the pro-apoptotic effect of miR-122, indicating that $\mathrm{XIAP}$ is a prominent target that mediates miR-122-mediated regulation of EC apoptosis (Fig. 3) [49]. Notably, miR-122 has been identified as a possible risk factor for recurrent cardiovascular events in ACS and cerebral ischemic events [50]. MiR-122 was found to mainly originate from circulating ECs and monocytes and was increased in patients with AMI compared with patients with unstable angina [51]. Thus, miR-122 may serve as a positive regulator of EC apoptosis induced by atherogenic factors, suggesting its use as a noninvasive diagnostic biomarker and potential treatment for therapeutic interventions for atherosclerosis, ACS and other atherosclerotic heart diseases.

\section{Roles of MiR-122 in Heart Failure}

Myocardial fibrosis and remodeling are the key causes of HF. Physiological, pharmacological and clinical studies have demonstrated that abnormal miRs and activation of the RAS are important mediators of the progression of HF, which is the end stage of cardiovascular disease and leads to systolic and/or diastolic dysfunction in the heart [2, 32, $47,52,53]$. These effects include blood stagnation in the venous system and insufficient perfusion in arteries in the cardiovascular system. Myocardial fibrosis was associated with higher long-term mortality in patients with $\operatorname{HF}[2,39]$. Recently, we demonstrated that Ang II triggered increased apoptosis and reduced of SIRT6, ELA and ACE2 levels in rat $\mathrm{AFs}$, which were exacerbated by the overexpression of miR-122-5p via miR-122-5p mimic but were prevented by administration of miR-122-5p inhibitor [1]. Notably, miR122 mimics significantly reversed the ELA-mediated beneficial effects on apoptosis and autophagy imbalance in rat AFs, accompanied with decreased AMPK phosphorylation and elevated mTOR phosphorylation level (Table 1; Fig. 3) [1]. In addition, pretreatment with apelin-13 inhibited Ang II-induced cardiac fibrosis in rats with HF by blocking collagen production and activating the TGF- $\beta$-CTGF signaling pathway [2, 54]. Importantly, the upregulation of ACE2 mediated by SIRT6 and apelin is a key mechanism by which the RAS, cardiovascular fibrosis and HF are inhibited [2, 44, 52, 53]. ACE2 is a negative receptor of RAS and a coreceptor for severe respiratory syndrome coronavirus 2 (SARSCoV2), which causes entry for the novel coronavirus disease 2019 (COVID-19) pneumonia [1, 39]. The major contributor to progressively worsened systemic manifestations of COVID-19 was due to imbalance of the Ang-(1-7)/Ang II through a loss of functional tissue ACE2, leading to severe inflammatory storm [39]. In pre-clinical models and clinical populations, recombinant human ACE2 use in patients with HF led to improvement of pathological hypertrophy, fibrosis, inflammation, oxidant injury and heart dysfunction, reflecting ACE2-mediated cardiac protective impacts [2, 39, $52,53]$. The apelin/ACE2 pathway has been implicated in hypertension and HF, making it a promising therapeutic target $[1,2,6,52,53]$. Recently, miR-122-5p was shown to be increased in a rat model of postinfarction HF, and the proapoptotic role of miR-122-5p was revealed [55]. Furthermore, in addition to its role as a clinical risk factor, miR-122 has been implicated as a prognostic biomarker and increases NT-proBNP level. An elevated level of miR-122 was significantly associated with right ventricular dysfunction and could predict all-cause and cardiovascular mortality, 
especially improving HF risk stratification of patients with reduced LVEF (Table.1) [21]. However, lower fluctuating serum miR-122-5p levels were observed in patients with acute HF, indicating that miR-122-5p may be linked with acute cardiac decompensation through treatment courses [54]. Thus, miR-122 may be an independent risk factor for the development of chronic and acute HF.

\section{Roles of MiR-122 in Myocardial Infarction}

Circulating miR-122-5p is considered a promising novel diagnostic biomarker for patients with acute MI [50, 56-59], as levels of miR-122-5p have been found to be obviously increased in patients with acute MI [56-59]. Circulating miR-122-5p expression was increased in AMI patients at 4, 8,12 , and $24 \mathrm{~h}$ after MI compared to non-AMI controls and displayed a trend similar to that of $\mathrm{cTnI}$ levels concentrations in AMI patients [56]. Ischemic cell death leads to the formation of damaged tissue in the heart, which is replaced with a fibrotic scar produced by fibroblasts and myofibroblasts, eventually resulting in scarring, cardiac fibrosis, and remodeling [2, 26-29]. Fibrosis during MI can be classified as reactive or replacement fibrosis. Importantly, reactive fibrosis at the infarct border zone and the remote uninjured myocardium, leads to chamber compliance alteration, increased ventricular stiffness and compromised cardiac output [22, 47]. Replacement fibrosis refers to scar formation, a critical process for preventing bursting of the ventricular wall after ischemic insult during MI $[22,60]$. In addition, an elevated ratio between circulating miR-122-5p/miR-133b is a specific early prognostic biomarker in acute MI [57]. Therefore, miR-122 is emerging as a prognostic biomarker of infarct size and cardiac dysfunction in MI. Overexpression of miR122 remarkably attenuated Lycium barbarum polysaccharide (LBP)-mediated protective activity in hypoxia-injured H9C2 cells, while the inhibition of miR-122 accelerated LBP-mediated protective activity [60]. Surprisingly, the LBP-induced activation of the phosphorylated extracellular signal-regulated kinase (ERK) and AMPK signaling pathways was attenuated by miR-122 overexpression and accelerated by miR-122 suppression [60]. An in vivo investigation revealed that, the administration of anti-miR-122 decreased infarct size and improved cardiac function in MI rats associated with improvements to the LVEF and left ventricular fractional shortening, indicating that miR-122 inhibition may have potential for the prevention and treatment of acute MI (Table 1; Fig. 3) [60]. Overexpression of miR-122 by miR-122 mimics inhibited cellular viability and promoted the apoptosis of CMs, while miR-122 knockdown promoted cell viability and inhibited apoptosis [61]. MiR-122-5p significantly reversed the beneficial effects of salvianolate on cardiac injury in rats with increased Bax and caspases- 3 expression and decreased of Bcl-2 expression [23]. The inhibition of miR-122 participates in the regulation of CM injury during oxygen and nutrient deprivation in acute MI through the activation of Akt/mTOR signaling and inactivation of the JNK/p38MAPK pathway. In contrast, the activation of miR-122 prevents cell survival and promotes cellular apoptosis during myocardial ischemia/reperfusion. Furthermore, miR-122 has been confirmed to be involved in cardiac repair, impairing epicardial expansion, and epithelial-mesenchymal transition through activation of the Akt/ GSK-3 $\beta / \beta$-catenin signaling pathway, eventually resulting in a rapid decline in cardiac function (Table 1; Fig. 3) [23, 24]. MiR-122 was highly expressed in the border zone of the ischemic myocardium in acute MI pigs without ventricular fibrillation compared with pigs in a sham group. MiR-122 was decreased in platelets and increased in PBMCs from the acute MI pigs [50]. These results suggest that miR-122 inhibition in controlling $\mathrm{CM}$ proliferation, apoptosis and cardiac dysfunction in MI.

\section{Roles of MiR-122 in Atrial Fibrillation}

MiR-122 has been shown to predict the risk of atrial fibrillation [25, 62]. Atrial fibrillation is associated with atrial structural, electrical, and contractile remodeling and atrial fibrosis, which is denoted by the deposition of ECM. Fibrous scarring and interstitial fibrosis in the heart decrease cardiac contractility, subsequently interfering with the normal electrical function of the heart, resulting to occurrence and development of arrhythmia [25, 63]. Surprisingly, compared with those in mice in sham-operated and control groups, miR-122 levels were significantly increased in male C57BL/6 mice with atrial fibrillation, and this increase was significantly decreased by transfection with the miR-122 inhibitor [63]. $\mathrm{CM}$ viability was increased and the $\mathrm{CM}$ apoptosis rate was significantly decreased following miR-122 transfection. In addition, miR-122 inhibition upregulated expression of the anti-apoptotic protein Bcl-x and downregulated expression of the pro-apoptotic protein caspase- 3 and the phosphorylation of ERK1/2 (Table 1; Fig. 3) [25], that implicating miR122 in the molecular mechanisms underlying the proliferation and apoptosis of CMs in atrial fibrillation.

\section{Conclusion}

MiR-122 appears to be a direct participant in the deteriorating cardiovascular system by promoting inflammation, oxidative stress, apoptosis and ECM deposition in various cardiovascular diseases. Clinical and experimental studies clearly support the physiological and pathophysiological roles of miR-122 in cardiovascular fibrosis and dysfunction. 
MiR-122 overexpression exacerbates the loss of autophagy and increased inflammation, apoptosis, extracellular matrix deposition, cardiovascular fibrosis and dysfunction mediated by Ang II. Most importantly, the inhibition of miR-122 can act as antifibrotic, anti-apoptotic, anti-inflammatory, antioxidant and proautophagic functions. Targeting the miR-122 has emerged as a prewarning biomarker and novel therapeutic approach against progression of cardiovascular fibrosis and related diseases and an increased understanding of cardiovascular actions of the miR-122 will help to develop effective interventions.

Acknowledgements This work was supported by the National Major Research Plan Training Program and General Program of the National Natural Science Foundation of China [81770253; 91849111 ; 81370362; 81300044] and Talent project of Beijing Chaoyang Hospital Affiliated to Capital Medical University.

\section{Compliance with Ethical Standards}

Conflict of interest All the authors declare that they have no conflict of interest.

\section{References}

1. Song, J. J., Yang, M., Liu, Y., Song, J. W., Wang, J., Chi, H. J., et al. (2020). MicroRNA-122 aggravates angiotensin II-mediated apoptosis and autophagy imbalance in rat aortic adventitial fibroblasts via the modulation of SIRT6-elabela-ACE2 signaling. European Journal of Pharmacology, 883, 173374. https://doi. org/10.1016/j.ejphar.2020.173374.

2. Ma, Z., Song, J. J., Martin, S., Yang, X. C., \& Zhong, J. C. (2020). The Elabela-APJ axis: A promising therapeutic target for heart failure. Heart Failure Reviews, 27, 1-10. https://doi.org/10.1007/ s10741-020-09957-5.

3. Pinar, A. A., Scott, T. E., Huuskes, B. M., Tapia Cáceres, F. E., Kemp-Harper, B. K., \& Samuel, C. S. (2020). Targeting the NLRP3 inflammasome to treat cardiovascular fibrosis. Pharmacology \& Therapeutics, 209, 107511.

4. Paul, M., Amos, Z., \& Wing-Kin, S. (2019). Fibrosis in chronic liver disease: An update on diagnostic and treatment modalities. Drugs, 79(9), 903-927.

5. Zhao, Z., Zhong, L., Li, P., He, K., Qiu, C., Zhao, L., et al. (2020). Cholesterol impairs hepatocyte lysosomal function causing M1 polarization of macrophages via exosomal miR-122-5p. Experimental Cell Research, 387(1), 111738.

6. Xu, R., Zhang, Z. Z., Chen, L. J., Yu, H. M., Guo, S. J., Xu, Y. L., et al. (2016). Ascending aortic adventitial remodeling and fibrosis are ameliorated with Apelin-13 in rats after TAC via suppression of the miRNA-122 and LGR4- $\beta$-catenin signaling. Peptides, 86 , $85-94$.

7. Filipowicz, W., \& Grosshans, H. (2011). The liver-specific microRNA miR-122: Biology and therapeutic potential. Progress in Drug Research, 67, 221-238.

8. Gatfield, D., Le Martelot, G., Vejnar, C. E., Gerlach, D., Schaad, O., Fleury-Olela, F., et al. (2009). Integration of microRNA miR122 in hepatic circadian gene expression. Genes \& Development, 23(11), 1313-1326.

9. Rivoli, L., Vliegenthart, A. D., de Potter, C. M., van Bragt, J. J., Tzoumas, N., Gallacher, P., et al. (2017). The effect of renal dysfunction and haemodialysis on circulating liver specific miR122. British Journal of Clinical Pharmacology, 83(3), 584-592.

10. Lunney, M., Ruospo, M., Natale, P., Quinn, R. R., Ronksley, P. E., Konstantinidis, I., et al. (2020). Pharmacological interventions for heart failure in people with chronic kidney disease. Cochrane Database System Review, 2(2), CD012466.

11. Song, J. J., Ma, Z., Wang, J., Chen, L. X., \& Zhong, J. C. (2020). Gender differences in hypertension. The Journal of Cardiovascular Translational Research, 13(1), 47-54.

12. Hu, J., Wu, H., Wang, D., Yang, Z., \& Dong, J. (2019). LncRNA ANRIL promotes NLRP3 inflammasome activation in uric acid nephropathy through miR-122-5p/BRCC3 axis. Biochimie, 157, 102-110.

13. Qu, X. H., \& Zhang, K. (2018). MiR-122 regulates cell apoptosis and ROS by targeting DJ-1 in renal ischemic reperfusion injury rat models. European Review for Medical and Pharmacological Sciences, 22(24), 8830-8838.

14. Wang, Y., Liang, H., Jin, F., Yan, X., Xu, G., Hu, H., et al. (2019). Injured liver-released miRNA-122 elicits acute pulmonary inflammation via activating alveolar macrophage TLR7 signaling pathway. Proceedings of the National academy of Sciences of the United States of America, 116(13), 6162-6171.

15. Hu, Y., Du, G., Li, G., Peng, X., Zhang, Z., \& Zhai, Y. (2019). The miR-122 inhibition alleviates lipid accumulation and inflammation in NAFLD cell model. Archives of Physiology and Biochemistry, 16, 1-5.

16. Snyder-Talkington, B. N., Dong, C., Sargent, L. M., Porter, D. W., Staska, L. M., Hubbs, A. F., et al. (2016). mRNAs and miRNAs in whole blood associated with lung hyperplasia, fibrosis, and bronchiolo-alveolar adenoma and adenocarcinoma after multi-walled carbon nanotube inhalation exposure in mice. Journal of Applied Toxicology, 36(1), 161-174.

17. Weber, G. J., Purkayastha, B., Ren, L., Pushpakumar, S., \& Sen, U. (2018). Hypertension exaggerates renovascular resistance via miR-122-associated stress response in aging. Journal of Hypertension, 36(11), 2226-2236.

18. Song, G., Zhu, L., Ruan, Z., Wang, R., \& Shen, Y. (2019). MicroRNA-122 promotes cardiomyocyte hypertrophy via targeting FoxO3. Biochemical and Biophysical Research Communications, 519(4), 682-688.

19. Zhang, H. G., Zhang, Q. J., Li, B. W., Li, L. H., Song, X. H., Xiong, C. M., et al. (2020). The circulating level of miR-122 is a potential risk factor for endothelial dysfunction in young patients with essential hypertension. Hypertension Research, 43(6), 511-517.

20. Martínez-Micaelo, N., Beltrán-Debón, R., Baiges, I., Faiges, M., \& Alegret, J. M. (2017). Specific circulating microRNA signature of bicuspid aortic valve disease. Journal of Translational Medicine, 15(1), 76.

21. Stojkovic, S., Koller, L., Sulzgruber, P., Hülsmann, M., Huber, K., Mayr, M., et al. (2020). Liver-specific microRNA-122 as prognostic biomarker in patients with chronic systolic heart failure. International Journal of Cardiology, 303, 80-85.

22. Ivey, M. J., Kuwabara, J. T., Pai, J. T., Moore, R. E., Sun, Z., \& Tallquist, M. D. (2018). Resident fibroblast expansion during cardiac growth and remodeling. Journal of Molecular and Cellular Cardiology, 114, 161-174.

23. Lin, J., \& Zheng, X. (2017). Salvianolate blocks apoptosis during myocardial infarction by downregulating miR-122-5p. Current Neurovascular Research, 14(4), 323-329.

24. Gong, L., Chang, H., \& Xu, H. (2019). LncRNA MALAT1 knockdown alleviates oxygen-glucose deprivation and reperfusion induced cardiomyocyte apoptotic death by regulating miR122. Experimental and Molecular Pathology, 111, 104325. 
25. Zhang, X., \& Jing, W. (2018). Upregulation of miR-122 is associated with cardiomyocyte apoptosis in atrial fibrillation. Molecular Medicine Reports, 18(2), 1745-1751.

26. González, A., Schelbert, E. B., Díez, J., \& Butler, J. (2018). Myocardial interstitial fibrosis in heart failure: Biological and translational perspectives. Journal of the American College of Cardiology, 71(15), 1696-1706.

27. Prabhat, R., Rajesh, K., \& Kumar, V. S. (2019). Cardiac fibroblasts and cardiac fibrosis: Precise role of exosomes. Frontiers in Cell and Developmental Biology, 7, 318.

28. Tallquist Michelle, D. (2020). Cardiac fibroblast diversity. Annual Review of Physiology, 82, 63-78.

29. Harvey, A., Montezano, A. C., Lopes, R. A., Rios, F., \& Touyz, R. M. (2016). Vascular fibrosis in aging and hypertension: Molecular mechanisms and clinical implications. Canadian Journal of Cardiology, 32(5), 659-668.

30. Lokugamage, N., Choudhuri, S., Davies, C., Chowdhury, I. H., \& Garg, N. J. (2020). Antigen-based nano-immunotherapy controls parasite persistence, inflammatory and oxidative stress, and cardiac fibrosis, the hallmarks of chronic chagas cardiomyopathy, in a mouse model of trypanosoma cruzi infection. Vaccines (Basel), 8(1), 96. https://doi.org/10.3390/vaccines8010096.

31. Gyöngyösi, M., Winkler, J., Ramos, I., Do, Q. T., Firat, H., McDonald, K., et al. (2017). Myocardial fibrosis: Biomedical research from bench to bedside. European Journal of Heart Failure, 19(2), 177-191.

32. Chen, L. J., Xu, R., Yu, H. M., Chang, Q., \& Zhong, J. C. (2015). The ACE2/apelin signaling, microRNAs, and hypertension. International Journal of Hypertension, 2015, 896861.

33. Zhang, H. N., Xu, Q. Q., Thakur, A., Alfred, M. O., Chakraborty, M., Ghosh, A., et al. (2018). Endothe-lial dysfunction in diabetes and hypertension: Role of microRNAs and long non-coding RNAs. Life Sciences, 213, 258-268.

34. Sygitowicz, G., Maciejak-Jastrzębska, A., \& Sitkiewicz, D. (2020). MicroRNAs in the development of left ventricular remodeling and postmyocardial infarction heart failure. Polish Archives of Internal Medicine, 130(1), 59-65. https://doi. org/10.20452/pamw.15137.

35. Liao, C. H., Wang, C. Y., Liu, K. H., Liu, Y. Y., Wen, M. S., \& Yeh, T. S. (2018). MiR-122 marks the differences between subcutaneous and visceral adiposetissuesand associates with the outcome of bariatric surgery. Obesity Research \& Clinical Practice, 12(6), 570-577.

36. Wang, Y., Jin, P., Liu, J., \& Xie, X. (2019). Exosomal microRNA-122 mediates obesity-related cardiomyopathy through suppressing mitochondrial ADP-ribosylation factor-like 2. Clinical Science, 133(17), 1871-1881.

37. Liang, W., Guo, J., Li, J., Bai, C., \& Dong, Y. (2016). Downregulation of miR-122 attenuates hypoxia/ reoxygenation (H/R)induced myocardial cell apoptosis by upregulating GATA-4. Biochemical and Biophysical Research Communications, 478(3), 1416-1422.

38. Zhang, X., Wang, X., Wu, J., Peng, J., Deng, X., Shen, Y., et al. (2018). The diagnostic values of circulating miRNAs for hypertension and bioinformatics analysis. Bioscience Reports, 38(4), BSR20180525.

39. Gheblawi, M., Wang, K., Viveiros, A., Nguyen, O., Zhong, J., Turner, A. J., et al. (2020). Angiotensin converting enzyme 2: SARS-CoV-2 receptor and regulator of the renin-angiotensin system-celebrating the 20th Anniversary of the Discovery of ACE2. Circulation Research, 126(10), 1456-1474.

40. Leimena, C., \& Qiu, H. (2018). Non-coding RNA in the pathogenesis, progression and treatment of hypertension. International Journal of Molecular Sciences, 19(4), 927. https://doi. org/10.3390/ijms 19040927.
41. Cengiz, M., Karatas, O. F., Koparir, E., Yavuzer, S., Ali, C., Yavuzer, H., et al. (2015). Differential expression of hypertensionassociated microRNAs in the plasma of patients with white coat hypertension. Medicine (Baltimore), 94(13), e693.

42. Xu, S. (2020). Therapeutic potential of blood flow mimetic compounds in preventing endothelial dysfunction and atherosclerosis. Pharmacological Research, 155, 104737.

43. Elhanati, S., Ben-Hamo, R., Kanfi, Y., Varvak, A., Glazz, R., Lerrer, B., et al. (2016). Reciprocal regulation between SIRT6 and miR-122 controls liver metabolism and predicts hepatocarcinoma prognosis. Cell Reports, 14(2), 234-242.

44. Zhang, Z. Z., Cheng, Y. W., Jin, H. Y., Chang, Q., Shang, Q. H., Xu, Y. L., et al. (2017). The sirtuin 6 prevents angiotensin II-mediated myocardial fibrosis and injury by targeting AMPKACE2 signaling. Oncotarget, 8(42), 72302-72314.

45. Zhou, X., Wang, J., Fa, Y., \& Ye, H. (2019). Signature microRNA expression profile is associated with spontaneous hypertension in African green monkey. Clinical and Experimental Hypertension, 41(3), 287-291.

46. Menendez-Castro, C., Cordasic, N., Dambietz, T., Veelken, R., Amann, K., Hartner, A., et al. (2020). Correlations between interleukin-11 expression and hypertensive kidney injury in a rat model of renovascular hypertension. American Journal of Hypertension, 33(4), 331-340.

47. Wang, J., Wei, L., Yang, X., \& Zhong, J. (2019). Roles of growth differentiation factor 15 in atherosclerosis and coronary artery disease. Journal of the American Heart Association, 8(17), e012826.

48. Wang, Y. L., \& Yu, W. (2018). Association of circulating microRNA-122 with presence and severity of atherosclerotic lesions. Peer J, 6, e5218.

49. Li, Y., Yang, N., Dong, B., Yang, J., Kou, L., \& Qin, Q. (2019). MicroRNA-122 promotes endothelial cell apoptosis by targeting XIAP: Therapeutic implication for atherosclerosis. Life Sciences, 232, 116590.

50. Barraclough, J. Y., Joan, M., Joglekar, M. V., Hardikar, A. A., \& Patel, S. (2019). MicroRNAs as prognostic markers in acute coronary syndrome patients-a systematic review. Cells, $8(12)$, $1572-1584$.

51. Li, X. D., Yang, Y. J., Wang, L. Y., Qiao, S. B., Lu, X. F., Wu, Y. J., et al. (2017). Elevated plasma miRNA-122, -140-3p, -720, -2861 , and -3149 during early period of acute coronary syndrome are derived from peripheral blood mononuclear cells. PLOS ONE, 12(9), e0184256.

52. Patel, V. B., Zhong, J. C., Grant, M. B., \& Oudit, G. Y. (2016). Role of the ACE2/angiotensin 1-7 axis of the renin-angiotensin system in heart failure. Circulation Research, 118(8), 1313-1326.

53. Zhang, Z. Z., Wang, W., Jin, H. Y., McKinnie, S. M., Farhan, M., Paul, M., et al. (2017). Pyr-apelin-13 is a negative regulator of angiotensin II-mediated adverse myocardial remodeling and dysfunction. Hypertension, 70(6), 1165-1175.

54. Koyama, S., Kuragaichi, T., Sato, Y., Kuwabara, Y., Usami, S., Horie, T., et al. (2017). Dynamic changes of serum microRNA$122-5 p$ through therapeutic courses indicates amelioration of acute liver injury accompanied by acute cardiac decompensation. ESC Heart Failure, 4(2), 112-121.

55. Liu, X., Meng, H., Jiang, C., Yang, S., Cui, F., \& Yang, P. (2016). Differential microRNA expression and regulation in the rat model of post-infarction heart failure. PLoS ONE, 11(8), e0160920.

56. Yao, X. L., Lu, X. L., Yan, C. Y., et al. (2015). Circulating miR$122-5 p$ as a potential novel biomarker for diagnosis of acute myocardial infarction. International Journal of Clinical and Experimental Pathology, 8(12), 16014-16019.

57. Cortez-Dias, N., Costa, M. C., Carrilho-Ferreira, P., Silva, D., Jorge, C., Calisto, C., et al. (2016). Circulating miR-122-5p/miR$133 \mathrm{~b}$ ratio is a specific early prognostic biomarker in acute myocardial infarction. Circulation Journal, 80(10), 2183-2191. 
58. Wang, Y., Chang, W., Zhang, Y., Zhang, L., Ding, H., Qi, H., et al. (2019). Circulating miR-22-5p and miR-122-5p are promising novel biomarkers for diagnosis of acute myocardial infarction. Journal of Cellular Physiology, 234(4), 4778-4786.

59. Fu, X., Khalil, H., Kanisicak, O., Boyer, J. G., Vagnozzi, R. J., Maliken, B. D., et al. (2018). Specialized fibroblast differentiated states underlie scar formation in the infarcted mouse heart. Journal of Clinical Investigation, 128(5), 2127-2143.

60. Li, Q., Zhang, Z., Li, H., Pan, X., Chen, S., Cui, Z., et al. (2019). Lycium barbarum polysaccharides protects $\mathrm{H} 9 \mathrm{c} 2$ cells from hypoxia-induced injury by down-regulation of miR-122. Biomedicine \& Pharmacotherapy, 110, 20-28.

61. Zhang, Z. W., Li, H., Chen, S. S., Li, Y., Cui, Z. Y., \& Ma, J. (2017). MicroRNA-122 regulates caspase- 8 and promotes the apoptosis of mouse cardiomyocytes. Brazilian Journal of Medical and Biological Research, 50(2), e5760.
62. Chen, C., Li, R., Ross, R. S., \& Manso, A. M. (2016). Integrins and integrin-related proteins in cardiac fibrosis. Journal of Molecular and Cellular Cardiology, 93, 162-174.

63. Zhang, Z., Li, H., Cui, Z., Zhou, Z., Chen, S., Ma, J., et al. (2019). Long non-coding RNA UCA1 relieves cardiomyocytes H9c2 injury aroused by oxygen-glucose deprivation via declining miR122. Artificial Cells, Nanomedicine, and Biotechnology, 47(1), 3492-3499.

Publisher's Note Springer Nature remains neutral with regard to jurisdictional claims in published maps and institutional affiliations. 